\title{
Targeting cancers in the gastrointestinal tract: role of capecitabine
}

\author{
Muhammad Wasif Saif \\ Yale Cancer Center, Yale University \\ School of Medicine, New Haven, CT, USA
}

\begin{abstract}
Capecitabine is currently the only novel, orally home-administered fluorouracil prodrug. It offers patients more freedom from hospital visits and less inconvenience and complications associated with infusion devices. The drug has been extensively studied in large clinical trials in many solid tumors, including breast cancer, colorectal cancer, gastric cancer, and many others. Furthermore, the drug compares favorably with fluorouracil in patients with such cancers, with a safe toxicity profile, consisting mainly of gastrointestinal and dermatologic adverse effects. Whereas gastrointestinal events and hand-foot syndrome occur often with capecitabine, the tolerability profile is comparatively favorable. Prompt recognition of severe adverse effects is the key to successful management of capecitabine. Ongoing and future clinical trials will continue to examine, and likely expand, the role of capecitabine as a single agent and/or in combination with other anticancer agents for the treatment of gastrointestinal as well as other solid tumors, both in the advanced palliative and adjuvant settings. The author summarizes the current data on the role of capecitabine in the management of gastrointestinal cancers.
\end{abstract}

Keywords: 5-fluorouracil, capecitabine, chemotherapy, adjuvant, advanced, colon cancer, gastric cancer, hepatocellular cancer, pancreatic cancer, cholangiocarcinoma, rectal cancer, anal cancer

\section{Introduction}

Fluoropyrimidines remain the standard treatment regimens for numerous types of solid tumors. Capecitabine (Xeloda ${ }^{\circledR}$, Hoffmann-La Roche Inc., Nutley, New Jersey, USA), a thymidine phosphorylase (TP)-activated fluoropyrimidine carbamate, is the only universally approved orally administered fluoropyrimidine. It belongs to a newer generation of orally administered fluoropyrimidines. The clinical need for convenient, tolerable and efficient agents that do not require continuous infusion like the original fluorinated analog of uracil, 5-fluorouracil (5-FU), prompted the development of capecitabine.

\section{Chemistry}

5-FU is not clinically useful when administered orally because extensive metabolism by dihydropyrimidine dehydrogenase (DPD) in the mucosa of the gastrointestinal tract and the liver leads to highly variable bioavailability. Capecitabine (Figure 1) is an oral prodrug of 5-FU that is absorbed intact through the intestinal wall and then converted to $5-\mathrm{FU}$ in three sequential enzymatic reactions. ${ }^{1}$ The final requisite enzyme, thymidine phosphorylase, is present at consistently higher levels in tumor rather than normal tissues, thereby suggesting that 5-FU delivered in this way may benefit from an element of tumor targeting and thus enhanced selectivity and better tolerability. ${ }^{2}$ Clinical evidence to support this comes from a study in patients with colorectal cancer. Capecitabine was administered 7 days before planned resection of the primary cancer and 5-FU levels assayed in tumor and adjacent tissues. 


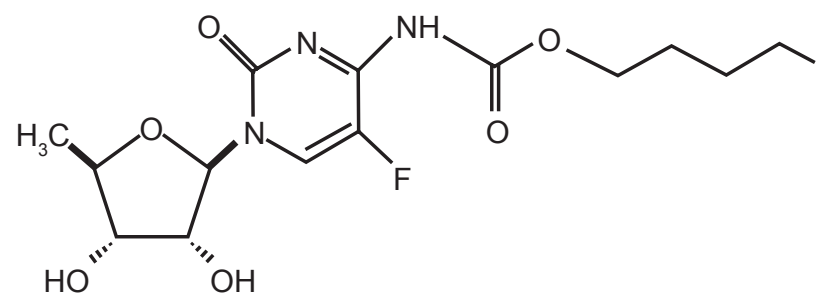

Capecitabine: 5'-deoxy-5-fluoro-N-[(pentyloxy) carbonyl]-cytidine. Molecular weight $=359.35$.

Figure I Structure of capecitabine.

The median ratio of 5-FU concentration in colorectal tumors to adjacent tissues was 2.9 (range $0.9-8.0$ ). ${ }^{3}$

\section{Clinical pharmacology}

Capecitabine is relatively non-cytotoxic in vitro and is readily absorbed from the gastrointestinal tract. In the liver, a $60 \mathrm{kDa}$ carboxyesterase hydrolyzes much of the compound to 5'-deoxy-5-fluorocytidine (5'-DFCR). Cytidine deaminase, an enzyme found in most tissues, including tumors, subsequently converts 5'-DFCR to 5'-deoxy-5-fluorouridine (5'-DFUR). The enzyme thymidine phosphorylase (dThdPase) then hydrolyzes 5'-DFUR to the active drug 5-FU. Many tissues throughout the body express thymidine phosphorylase ${ }^{4}$ and some human carcinomas including adenocarcinomas of the pancreas express this enzyme in higher concentrations than surrounding normal tissues. ${ }^{5}$

Both normal and tumor cells metabolize 5-FU to 5-fluoro-2-deoxyuridine monophosphate (FdUMP) and 5-fluorouridine triphosphate (FUTP). These metabolites cause cell injury by two different mechanisms. Firstly FdUMP and the folate cofactor, N5-10-methylenetetrahydrofolate, bind to thymidylate synthase (TS) to form a covalently bound ternary complex. This binding inhibits the formation of thymidylate from 2'-deaxyuridylate. Thymidylate is the necessary precursor of thymidine triphosphate, which is essential for the synthesis of DNA, so that a deficiency of this compound can inhibit cell division. Secondly nuclear transcriptional enzymes can mistakenly incorporate FUTP in place of uridine triphosphate (UTP) during the synthesis of RNA. This metabolic error can interfere with RNA processing and therefore protein synthesis.

\section{Pharmacokinetics and metabolism}

The pharmacokinetics of capecitabine and its metabolites have been evaluated in a number of studies over a dosage range of 500 to $3500 \mathrm{mg} / \mathrm{m}^{2} /$ day. ${ }^{3}$ Over this range the pharmacokinetics of capecitabine and its metabolite, 5'-DFCR, were linear and did not alter with time. The increases in the areas under curves (AUCs) of 5'-DFUR and 5-FU, however, were greater than proportional to the increase in dose and the AUC of 5-FU was $34 \%$ higher on day 14 than on day 1 . The elimination half-life of both parent capecitabine and 5-FU was about 45 minutes. The inter-patient variability in the $\mathrm{C}_{\max }$ and AUC of 5-FU was greater than $85 \%$. Capecitabine reached peak blood levels $\left(\mathrm{T}_{\max }\right)$ in about 1.5 hours with peak 5-FU levels occurring slightly later, at 2 hours. Food reduced both the rate and extent of absorption of capecitabine with mean $\mathrm{C}_{\max }$ and AUC decreased by $60 \%$ and $35 \%$, respectively. The $\mathrm{C}_{\text {max }}$ and AUC of 5-FU were also reduced by food by $43 \%$ and $21 \%$, respectively. Food delayed $\mathrm{T}_{\max }$ of both parent and 5-FU by 1.5 hours. Plasma protein binding of capecitabine and its metabolites is less than $60 \%$ and is not concentration-dependent. Capecitabine was primarily bound to human albumin (approximately 35\%).

Capecitabine is extensively metabolized enzymatically to 5-FU (Figure 2). The enzyme dihydropyrimidine dehydrogenase (DPD) hydrogenates 5-FU to the much less toxic 5-fluoro-5,6-dihydro-fluorouracil (FUH2). Dihydropyrimidinase cleaves the pyrimidine ring to yield 5-fluoro-ureido-propionic acid (FUPA). Finally beta-ureido-propionase cleaves FUPA to alfa-fluoro-beta-alanine (FBAL) which is cleared in the urine. Capecitabine and its metabolites are predominantly excreted in urine; $95.5 \%$ of administered capecitabine dose is recovered in urine. ${ }^{6}$ Fecal excretion is minimal $(2.6 \%)$. The major metabolite excreted in urine is FBAL which represents $57 \%$ of the administered dose. About $3 \%$ of the administered dose is excreted in urine as unchanged drug.

Capecitabine has been evaluated in patients with mild to moderate hepatic dysfunction due to liver metastases defined by a composite score including serum levels of bilirubin, aspartate and alanine transaminases and alkaline phosphatase. ${ }^{7}$ Following a single $1255 \mathrm{mg} / \mathrm{m}^{2}$ dose of capecitabine both AUC and $\mathrm{C}_{\text {max }}$ of capecitabine increased by $60 \%$ compared to patients with normal hepatic function $(n=14)$. Although the AUC and $\mathrm{C}_{\text {max }}$ of 5-FU was not affected capecitabine should be administered with caution in patients with even mild hepatic dysfunction and avoided in the presence of more severe liver impairment. There does not appear to be an effect on the pharmacokinetics of capecitabine or 5-FU with declining creatinine clearance but levels of 5-DFUR and FBAL do rise significantly once the creatinine clearance falls by more than $50 \%{ }^{8}$

\section{Drug interactions}

In vitro enzymatic studies with human liver microsomes indicated that capecitabine and 5'-DFUR had no inhibitory effects 

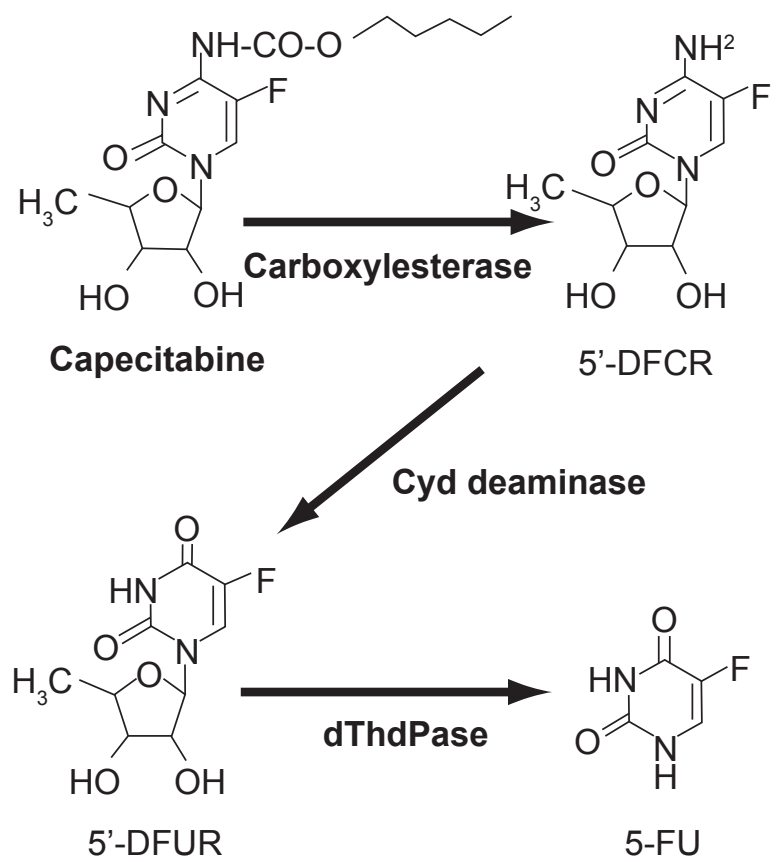

5'-DFCR = 5'-deoxy-5-fluorocytidine; Cyt deaminase = cytidine deaminase; 5 '-DFUR = 5'-deoxy-5-fluorouridine; dThdPase $=$ thymidine phosphorylase; 5 -FU $=5$-fluorouracil.

Figure 2 Metabolism of capecitabine to 5-fluorouracil.

on substrates of cytochrome P450, suggesting a low likelihood of interactions with drugs metabolized by cytochrome P450 enzymes. However an important interaction exists between capecitabine and warfarin, resulting in exaggerated anticoagulant activity and requiring close monitoring of the INR if these drugs are co-administered. ${ }^{9}$ The exact mechanism of the interaction is uncertain. An additional interaction occurred when Maalox $^{\circledR}(20 \mathrm{~mL})$, an aluminium hydroxide- and magnesium hydroxide-containing antacid, was administered immediately after capecitabine $\left(1250 \mathrm{mg} / \mathrm{m}^{2}\right)$. AUC and $\mathrm{C}_{\max }$ increased by $16 \%$ and $35 \%$, respectively for capecitabine and by $18 \%$ and $22 \%$, respectively, for 5 '-DFCR. No effect was observed on the other three major metabolites ( 5 '-DFUR, 5-FU, FBAL) of capecitabine. ${ }^{10}$

\section{Summary of safety and tolerability}

Capecitabine is relatively non-cytotoxic in vivo and thus the toxicity profile reflects that of its active metabolite 5-FU. The toxicity of 5-FU is known to be schedule-dependent. Bolus 5-FU causes mainly diarrhea, oral mucositis, myelosuppression and ocular irritation while infusional schedules may cause hand-foot syndrome (HFS) and ulcers on the lips but rarely result in significant myelosuppression. Interestingly the toxicity of capecitabine lies somewhere between that of bolus and infusional 5-FU. ${ }^{11}$ Diarrhea occurs in up to $50 \%$ of patients and may be severe, requiring hospital admission for intravenous (iv) fluids and antibiotics if accompanied by neutropenia. About half the patients treated will also develop a degree of HFS which may be helped by the use of pyridoxine (vitamin B6). Many patients indicate that fatigue is often the most disruptive side effect of all and this may continue for some time after treatment has ended. Diarrhea occurs in around half the patients who receive capecitabine. This can be quite severe but is usually well controlled with medication. Mouth ulcers may occur and nausea and/or vomiting affect $30 \%$ of patients but is readily controlled with antiemetics. Occasional side effects include constipation, headaches, conjunctivitis, anorexia, abdominal pain, hair thinning, ankle swelling and chest pain due to coronary vasospasm. $^{12}$

\section{Clinical activity}

Capecitabine is currently approved by the FDA for use as first-line therapy in patients with metastatic colorectal cancer when single-agent fluoropyrimidine therapy is preferred. The drug is also approved for use as a single agent in metastatic breast cancer patients who are resistant to both anthracyclineand paclitaxel-based regimens or in whom further anthracycline treatment is contra-indicated and in combination with docetaxel after failure of prior anthracycline-based chemotherapy. ${ }^{13}$ Single-agent and combination regimens have also shown benefits in patients with prostate, pancreatic, renal cell, and ovarian cancers. Improved tolerability and comparable efficacy compared with iv 5-FU/LV (5-FU with leucovorin) in addition to oral administration make capecitabine an attractive option for the treatment of several types of cancers as well as the focus of future trials.

\section{Colorectal cancer}

For more than 40 years, 5 -FU has been the foundation treatment for metastatic colorectal cancer and has been used with LV or in combination with irinotecan or oxaliplatin. As first-line therapy in colon cancer patients capecitabine has been studied either as single-agent administration compared directly to iv 5-FU/LV in two large randomized phase III trials ${ }^{14}$ or in combination with irinotecan ${ }^{15,16}$ and oxaliplatin ${ }^{17-20}$ in non-comparative studies. In the adjuvant setting a large randomized phase III trial (X-ACT study), enrolling 1987 Dukes C colon cancer patients, was conducted comparing single-agent capecitabine administration with iv 5-FU/LV (Mayo Clinic regimen). ${ }^{21-23}$

In the first-line monotherapy setting, the two randomized, prospective phase III trials enrolled a total number of 1207 patients, who were randomized to receive either oral 
capecitabine $\left(1250 \mathrm{mg} / \mathrm{m}^{2}\right.$ bid 2 weeks on/1 week off in 3-week cycles) or the Mayo Clinic regimen ( $\mathrm{LV} 20 \mathrm{mg} / \mathrm{m}^{2}$ followed by 5 -FU $425 \mathrm{mg} / \mathrm{m}^{2}$ iv bolus on days $1-5$ in a 4-week cycle) ${ }^{24,25}$ (Figure 3). Capecitabine demonstrated a statistically significant superior objective response rate (ORR) compared with 5-FU/LV (26\% vs 17\%; p < 0.0002), even in a patient subpopulation with poor prognostic parameters. Time to progression (TTP) was equivalent in both arms (4.6 vs 4.7 months; $p=0.85$ ) and the same was revealed for overall survival (OS) (12.9 vs 12.8 months; $\mathrm{p}=0.48) .{ }^{51}$ As far as the safety profile was concerned, all-grade AEs, including diarrhea $(47.7 \%$ vs $58 \%$; p $<0.001)$, stomatitis ( 24.3 vs $61.6 \%$; p < 0.001), nausea (87.9 vs $47.6 \%$; p $<0.001$ ), alopecia ( 5 vs $20.6 \%$; $<<0.001$ ) and neutropenia requiring medical intervention $(1.2 \%$ vs $10.5 \% ; p<0.001)$ occurred significantly more frequently in the 5-FU/LV arm. HFS was the only AE occurring in significantly higher incidence with capecitabine $(55.5 \%$ vs $62 \%$; $<<0.001)$. In addition, grade $3 / 4$ stomatitis $(2 \%$ vs $14.7 \% ; \mathrm{p}<0.001)$ and grade $3 / 4$ neutropenia $(2.3 \%$ vs $22.8 \%$; $<<0.001)$ resulting in a significantly higher incidence of neutropenic fever and sepsis $(0.2 \%$ vs $3.4 \%, p<0.001)$, were more frequently observed with 5-FU/LV, whereas grade 3 HFS (17.1 vs $0.7 \%$ ) occurred more frequently with capecitabine. Grade 3 hyperbilirubinemia appeared more frequently $(18.3 \%$ vs $3.3 \%$; $p<0.001)$ in the capecitabine group but tended to be an isolated phenomenon involving only indirect bilirubin. Recently, a meta-analysis of the medical resource used in one of the trials demonstrated that single-agent capecitabine as monotherapy treatment for advanced, metastatic colorectal cancer, apart from being efficient and more tolerable than 5-FU/LV, also led to substantial reduction in medical resource use. ${ }^{26}$

Capecitabine has been also evaluated as first-line treatment in metastatic colorectal cancer patients in combination either with oxaliplatin ${ }^{17-20}$ or with irinotecan ${ }^{15,16}$ in non-comparative phase II studies. When administered in combination with oxaliplatin $\left(120 \mathrm{mg} / \mathrm{m}^{2}\right.$ on day 1 or $70 \mathrm{mg} / \mathrm{m}^{2}$ on days 1 and 8), the dose of capecitabine varied between $750 \mathrm{mg} / \mathrm{m}^{2}$ bid and $1250 \mathrm{mg} / \mathrm{m}^{2}$ on days $1-14$ followed by 7 days rest in a 3-week cycle. ${ }^{19-22}$ Capecitabine has been also evaluated as first-line treatment in metastatic colorectal cancer patients in combination either with oxaliplatin ${ }^{15-19}$ or with irinotecan ${ }^{19-22}$ in phase II-III studies. When administered in combination with oxaliplatin $\left(130 \mathrm{mg} / \mathrm{m}^{2}\right.$ on day 1 or $70 \mathrm{mg} / \mathrm{m}^{2}$ on days 1 and 8), the dose of capecitabine varied between $750 \mathrm{mg} / \mathrm{m}^{2}$ bid and $1250 \mathrm{mg} / \mathrm{m}^{2}$ on days $1-14$ followed by 7 days rest in 3-week cycle. ${ }^{19-20}$ Cassidy et al reported a two-arm, randomized, noninferiority, phase III study (XELOX-1; NO16966A) comparing XELOX with FOLFOX-4 in the first-line treatment of MCRC. ${ }^{27}$ After the pivotal phase III data for bevacizumab became available ${ }^{28}$ the protocol was amended to a randomized, $2 \times 2$ factorial design with two coprimary objectives. The intent-to-treat population comprised 634 patients from the original two-arm portion of the study, plus an additional 1400 patients after the start of the amended $2 \times 2$ design, for a total of 2034 patients. The median PFS was 8.0 months in

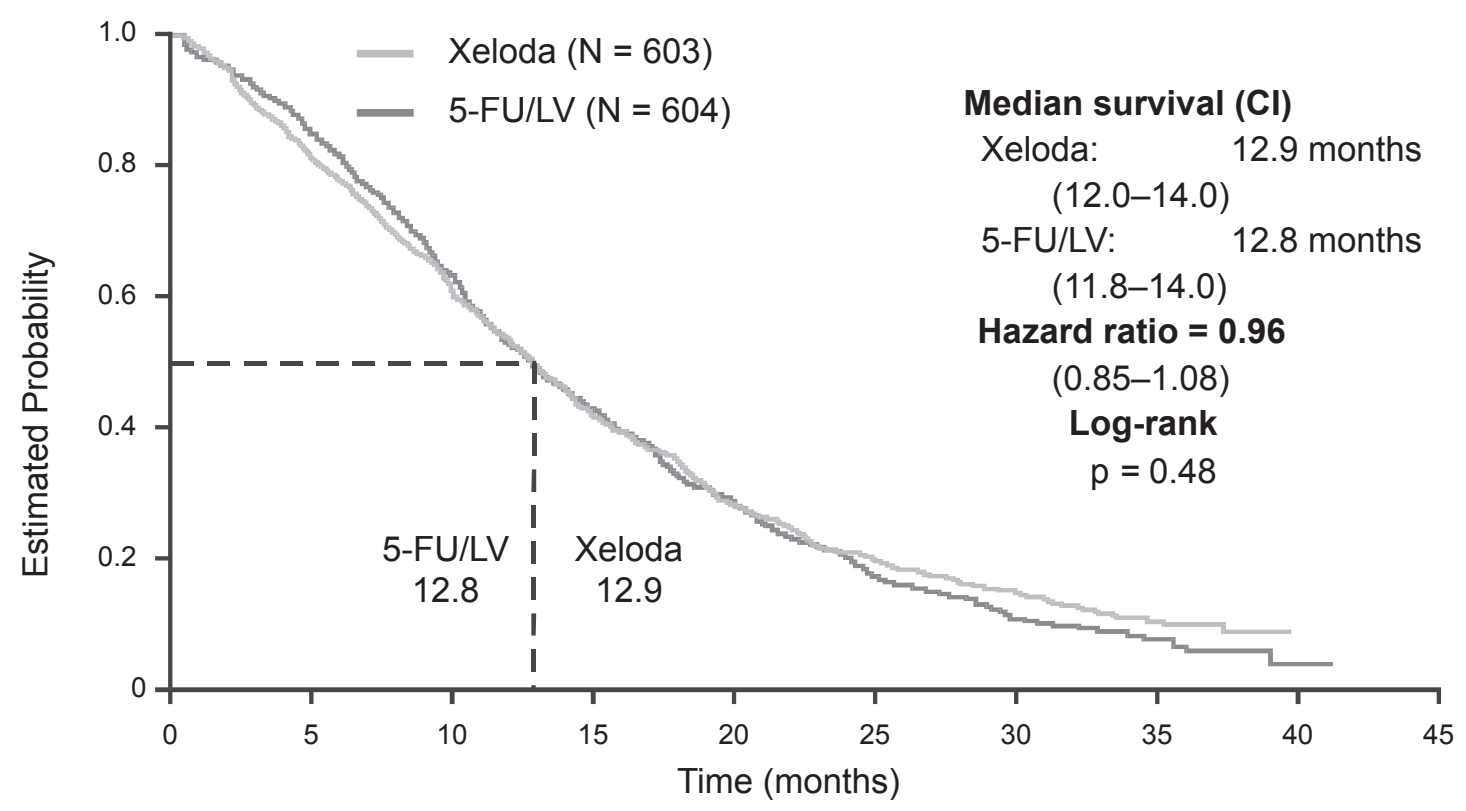

Figure 3 Xeloda vs 5-FU/LV integrated analysis equivalent survival with Xeloda and 5-FU/LV in metastatic CRC. Adapted with permission from Twelves C; Xeloda Colorectal Cancer Group. Capecitabine as first-line treatment in colorectal cancer. Pooled data from two large, phase III trails. Eur J Cancer. 2002;38 Suppl 2:I5-20.24 Copyright (C 2002 Elsevier. 
the pooled XELOX-containing arms versus 8.5 months in the FOLFOX-4-containing arms (hazard ratio [HR], 1.04; 97.5\% CI, 0.93-1.16). The median overall survival was 19.8 months with XELOX versus 19.6 months with FOLFOX-4 (HR, 0.99; 97.5\% CI, 0.88-1.12). FOLFOX-4 was associated with more grade 3/4 neutropenia/granulocytopenia and febrile neutropenia than XELOX, and XELOX with more grade 3 diarrhea and grade 3 hand-foot syndrome than FOLFOX-4. ${ }^{28}$ The main reasons for discontinuation were diarrhea ${ }^{28}$ and sensory neuropathy. In only one study did the initial dose of capecitabine have to be modified from $1000 \mathrm{mg} / \mathrm{m}^{2}$ bid to $750 \mathrm{mg} / \mathrm{m}^{2} \mathrm{bid}$, because of the high incidence $(85 \%)$ of diarrhea.

When administered concurrently with irinotecan, the dose of capecitabine ranged from $1000 \mathrm{mg} / \mathrm{m}^{2}$ to $1250 \mathrm{mg} / \mathrm{m}^{2} \mathrm{bid}$ on days 1-14 followed by 1 week rest for 21 days, while irinotecan was delivered at dose $240 \mathrm{mg} / \mathrm{m}^{2}$ to $300 \mathrm{mg} / \mathrm{m}^{2}$ on day 1 or $100 \mathrm{mg} / \mathrm{m}^{2}$ to $150 \mathrm{mg} / \mathrm{m}^{2}$ on days 1 and 8 in 3-week cycles. ${ }^{15,16,29,30}$ In the study conducted by Bajetta et $\mathrm{al}^{16}$ (capecitabine $1250 \mathrm{mg} / \mathrm{m}^{2}$ bid days $1-14$ and irinotecan $300 \mathrm{mg} / \mathrm{m}^{2}$ day 1 or $150 \mathrm{mg} / \mathrm{m}^{2}$ days 1,8 in 3 -week cycles) initial dose of both agents had to be modified to a lower dose (capecitabine $1000 \mathrm{mg} / \mathrm{m}^{2}$ bid and irinotecan $240 \mathrm{mg} / \mathrm{m}^{2}$ day 1 or $120 \mathrm{mg} / \mathrm{m}^{2}$ days 1,8 ) because of the increased incidence $(>33 \%)$ of grade $3 / 4$ diarrhea. Recently, a phase I/II pharmacokinetic study has demonstrated that the maximum tolerated dose of capecitabine is $1000 \mathrm{mg} / \mathrm{m}^{2} \mathrm{bid}$ days 1-14 every 3 weeks, when concurrently administered with irinotecan in chemo-naïve colorectal cancer patients. The recommended dose of the latter is $250 \mathrm{mg} / \mathrm{m}^{2}$ on day $1 .{ }^{31}$ The ORR in all studies ranged from $42 \%$ to $49 \%$, which is similar to the results previously reported with FOLFIRI. ${ }^{30,32}$ The most common grade $3 / 4$ AEs were diarrhea (19\%-27\%), neutropenia (11\%-12\%) and nausea/vomiting (10\%-12\%). In all studies, the incidence of grade 3 HFS secondary to capecitabine was lower compared with the one experienced with capecitabine alone. Although no comparative trial was performed, grade 3/4 neutropenia occurred less frequently (11\%-12\% vs $46 \%$ ) than with FOLFIRI, whereas the incidence of grade 3/4 diarrhea was higher. Both capecitabine combinations either with oxaliplatin or with irinotecan revealed comparable efficacy with FOLFOX4 and FOLFIRI, respectively, with acceptable safety and tolerability. The results of ongoing randomized comparative phase III trials will ascertain the future of these combinations in the first-line treatment of colorectal cancer.

In the adjuvant setting, capecitabine has been administered either as monotherapy or in combination with oxaliplatin. In a randomized, multicenter, comparative phase III trial (X-ACT) 1987 Dukes C resected colon cancer patients were randomized to receive as adjuvant treatment either single-agent capecitabine $\left(1250 \mathrm{mg} / \mathrm{m}^{2}\right.$ bid days $1-14$ every 21 days $[n=1004])$ or the Mayo Clinic regimen (LV $20 \mathrm{mg} / \mathrm{m}^{2}$ iv followed by bolus iv administration of 5-FU, $425 \mathrm{mg} / \mathrm{m}^{2}$ on days $1-5$ every 28 days, $[\mathrm{n}=983]$ ) over a period of 24 weeks. ${ }^{33}$ The X-ACT study demonstrated a significantly superior relapse-free survival $(p=0.053)$ and trends toward superior disease-free survival $(\mathrm{p}=0.053)$ and $\operatorname{OS}(\mathrm{p}=0.071)$ for the capecitabine arm (Figure 4). In addition, a positive safety profile was observed with capecitabine, producing significantly less of all grades of diarrhea (46 vs 64\%; p < 0.001), nausea/vomiting (36\% vs $51 \%$; p $<0.001)$, stomatitis $(22 \%$ vs $60 \%$; p $<0.001)$, alopecia (6\% vs $22 \%$; p $<0.001)$ and neutropenia (32 vs $63 \% ; \mathrm{p}<0.001$ ) (Figure 5). HFS was more frequently observed $(60 \%$ vs $9 \% ; \mathrm{p}<0.001)$ with capecitabine. In addition, grade $3 / 4$ neutropenia $(2 \%$ vs $26 \%$; $<0.001)$ leading to febrile neutropenia and consequently to sepsis $(0.3 \%$ vs $3 \%$; p $<0.001)$, and stomatitis, ( $2 \%$ vs $14 \%$; $\mathrm{p}<0.001)$ were significantly more common in the 5-FU arm. Grade 3 hyperbilirubinemia ( $\geq 3$ times the upper limit of normal) was more common with capecitabine (18\% vs $5.9 \%$ ). The incidence of grade $3 / 4$ abnormalities of the hepatic enzymes (serum glutamic-oxoacetic transaminase, serum glutamate-pyruvate transaminase), however, was low in both treatment arms $(0.7 \%$ and $1.6 \%$, respectively, with capecitabine, and $0.3 \%$ and $0.6 \%$, respectively, with 5-FU/LV).

The safety profile of capecitabine was similar, regardless of patients' age ( $<65$ or $\geq 65$ years of age). The incidence of first and second dose reduction was higher in patients receiving 5-FU/LV (42\% and 13\%, respectively, for capecitabine, and $44 \%$ and $26 \%$, respectively, for the latter). In addition, median time to first and second dose reduction was longer with capecitabine, compared with 5-FU/LV (78 and 113 days, respectively, for capecitabine, and 41 and 57 days, respectively, with $5-\mathrm{FU} / \mathrm{LV})$. Premature withdrawal was infrequent in both treatment arms (16 vs $12 \%$ ). These results led the FDA to approve capecitabine as single-agent adjuvant therapy for Dukes $\mathrm{C}$ colon cancer patients, when single fluoropyrimidine therapy is preferred.

In another randomized phase III trial in Dukes $\mathrm{C}$ colon cancer patients, three arms of adjuvant chemotherapy were compared: XELOX (capecitabine at dose $1000 \mathrm{mg} / \mathrm{m}^{2}$ bid days $1-14$ plus oxaliplatin $130 \mathrm{mg} / \mathrm{m}^{2}$ on day 1 , every 21 days for 8 cycles); Mayo Clinic regimen (LV $20 \mathrm{mg} / \mathrm{m}^{2}$ iv bolus 


\section{Disease-free survival}

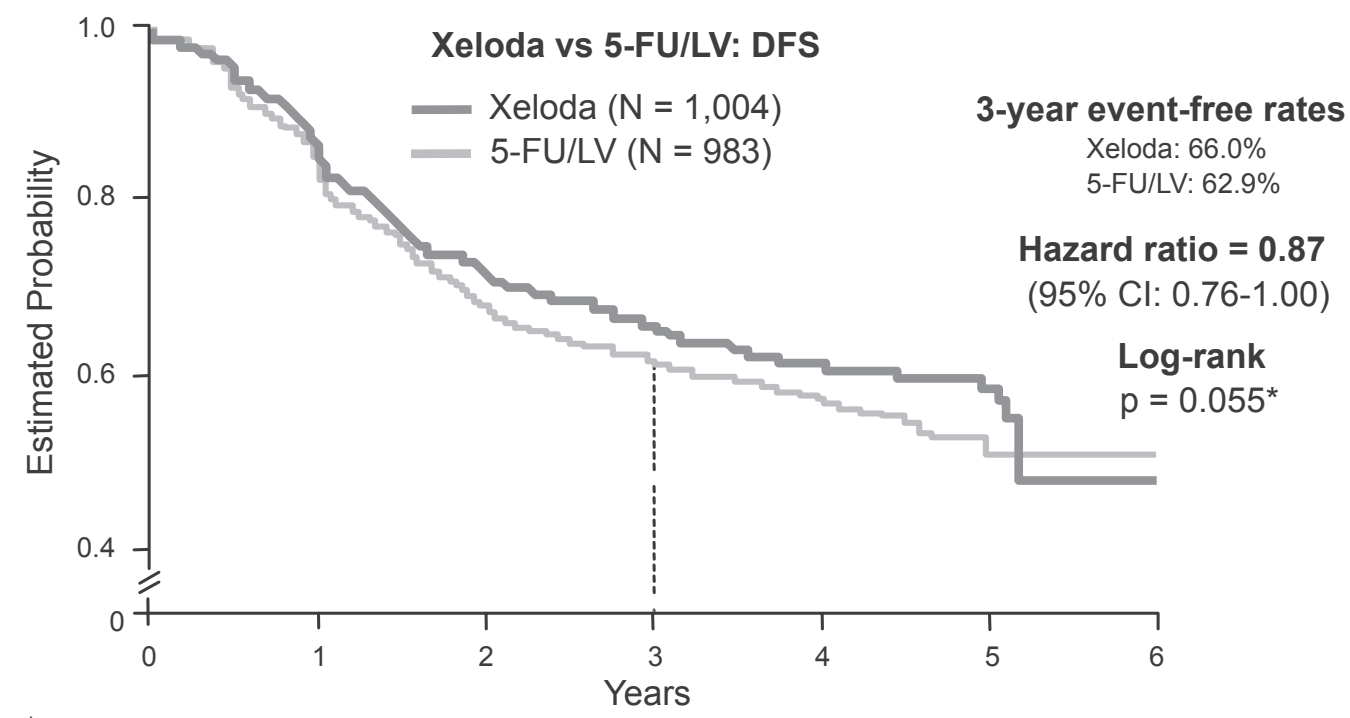

*Statistical test for superiority.

Figure 4 X-ACT:Adjuvant Xeloda vs 5-FU/LV.Adapted from Twelves C, et al. N Engl J Med. 2005;352:2696-2704.23

plus 5 -FU $425 \mathrm{mg} / \mathrm{m}^{2}$ iv bolus on days $1-5$, every 28 days for 6 cycles); and Roswell Park regimen (LV $500 \mathrm{mg} / \mathrm{m}^{2}$ iv plus iv 5 -FU $500 \mathrm{mg} / \mathrm{m}^{2}$ on day 1 , weeks $1-6$, in four 8-week cycles). Early safety data were presented at the American
Society of Clinical Oncology 2005 meeting. ${ }^{34}$ XELOX compared favorably with the other arms of the study. Grade $3 / 4$ neutropenia (5.3 vs 14 vs $3 \%$ respectively), febrile neutropenia ( $0.2 \%$ vs $4.7 \%$ vs $1.7 \%$ respectively), and also

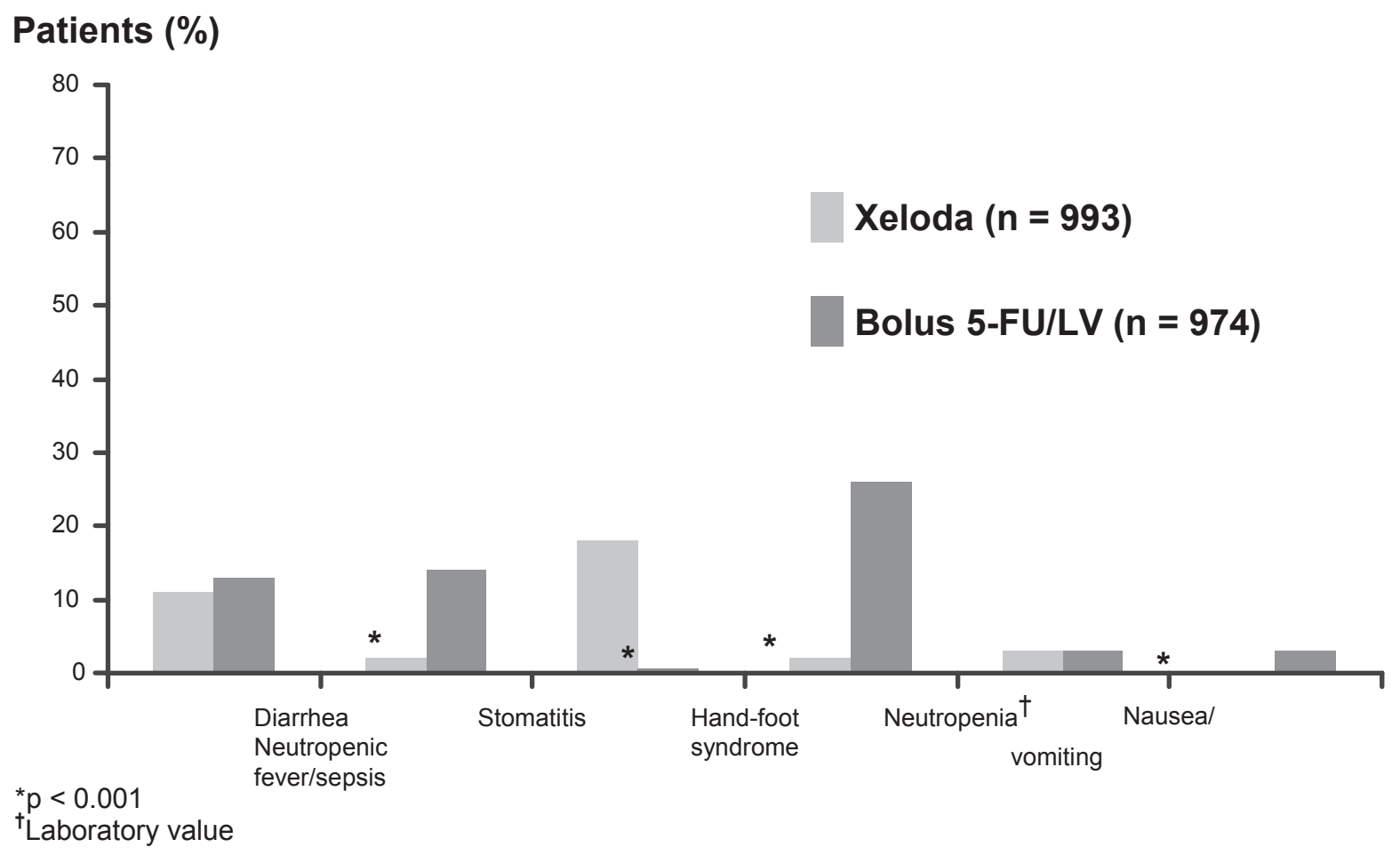

Figure 5 Adjuvant Xeloda: safety profile versus bolus 5-FU/LV (grade 3/4). Adapted from Scheithauer W, McKendrick J, Begdie S, et al. Oral capecitabine as an alternative to iv 5-FU-based adjuvant therapy for colon cancer: safety results of a randomized phase III trial. Ann Oncol. 2003; 14: 1735-1743. By permission of Oxford University Press. 
stomatitis ( $0.6 \%$ vs $11.2 \%$ vs $0 \%$, respectively) were less frequently observed with XELOX than with other treatment arms. Grade 3 HFS (3.6\% vs $0.2 \%$ vs $0.2 \%$, respectively), sensory neuropathy ( $8.1 \%$ vs 0 vs $0 \%$ ), and vomiting ( $5 \%$ vs $1.7 \%$ vs $4.6 \%$ ) occurred more frequently with XELOX compared with the other two arms. The incidence of grade 3/4 diarrhea was approximately the same with XELOX and the Mayo Clinic regimen (15\% vs $13.5 \%$, respectively), but higher with Roswell Park regimen (26.2\%). Early safety results of XELOX administration are comparable with those of the FOLFOX regimen, the standard adjuvant therapy for colon cancer patients. ${ }^{35}$ Efficacy results will be available in 2008 .

The proven efficacy and safety benefits of capecitabine over iv 5-FU/LV in the treatment of metastatic and early stage colon cancer have led to the development of a number of studies evaluating the use of capecitabine in chemoradiation schedules for patients with locally advanced rectal cancer (LARC) as single agent as well as in combination with oxaliplatin or irinotecan. Although several dosing and schedule protocols of single-agent capecitabine combined with radiotherapy for locally advanced rectal cancer patients have been evaluated in several noncomparative phase II trials, ${ }^{36-40}$ the continuous administration of capecitabine at a dose of $825 \mathrm{mg} / \mathrm{m}^{2}$ bid concurrently with radiotherapy was the most applied. We retrospectively compared the safety and efficacy of capecitabine-based regimens with well-established CIV-5-FU-based (continuous intravenous infusion 5-FU) regimens in LARC. ${ }^{41}$ We collected published data on 542 patients treated on either CIV-5-FU (197) or capecitabine (345) with concurrent radiation (external radiation treatment, XRT) for LARC. This included phase I or II studies published or available from Pubmed. Safety was assessed by determining proportion of patients who experienced grade III/IV adverse effects. Efficacy was assessed by determining pathological complete response (pCR). Chi-square tests were used to compare the two regimens. A p value less than 0.05 was considered statistically significant. Statistical tests were further corrected for multiplicity using the method of Benjamini and Yekutieli. We found that $\mathrm{pCR}$ was significantly higher in patients getting capecitabine vs CIV-5-FU (25 vs 13\%; p = 0.008, P [adj] = $0.034)$. Both regimens were generally well tolerated. There was no grade IV toxicity reported. Grade III HFS was more common in the capecitabine group, and grade III diarrhea was more common in the CIV group. Capecitabine appears to be tolerable, with no reported grade 4 AEs in most studies, and low incidence of grade 3 toxicities. Additionally, it is more convenient than protracted infusion of 5-FU and demonstrates comparable efficacy achieving satisfactory tumor down-staging rates and pathological response rates. The same observation was made for concurrent administration of XELOX and XELIRI with radiotherapy. ${ }^{42-47}$ In light of the encouraging results, two large randomized phase III trials are in progress. The National Surgical Adjuvant Breast and Bowel Project R-04 trial is recruiting patients to receive either radiotherapy plus capecitabine \pm oxaliplatin or plus infusional 5-FU \pm oxaliplatin. Following surgery, patients will be administered FOLFOX \pm bevacizumab. The Pan-European Trials in Adjuvant Colorectal Cancer 6 trial compares neoadjuvant capecitabine plus radiation followed by adjuvant capecitabine with or without oxaliplatin.

\section{Gastric or gastroesophageal cancer}

Capecitabine is being investigated in phase I-III trials for the treatment of gastric, gastroesophageal, and esophageal cancers, both in the first-line metastatic setting as well as in the adjuvant setting. The most frequently investigated combinations include capecitabine with docetaxel, paclitaxel, cisplatin, or oxaliplatin, and irinotecan. These therapies have yielded efficacy data that compare favorably with data from phase III trials of parenteral 5-FU in the first-line metastatic setting, and they mostly are well tolerated.

A phase III study evaluated XP (capecitabine/cisplatin) vs FP (5-FU/cisplatin) in first-line in patients with previously untreated measurable advanced gastric cancer. ${ }^{48}$ The patients received either oral capecitabine $\left(1000 \mathrm{mg} / \mathrm{m}^{2}\right.$ bid days $1-14)$ + cisplatin $\left(80 \mathrm{mg} / \mathrm{m}^{2}\right.$ iv day 1$) \mathrm{q} 3 \mathrm{w}$ (XP arm) or 5-FU $\left(800 \mathrm{mg} / \mathrm{m}^{2} /\right.$ day continuous infusion, days $\left.1-5\right)+$ cisplatin $\left(80 \mathrm{mg} / \mathrm{m}^{2}\right.$ iv day 1$) \mathrm{q} 3 \mathrm{w}$ (FP arm). XP requires 1 day per 3 weeks in hospital; FP requires 5 days. Patients were treated until disease progression or unacceptable toxicity. Primary endpoint was non-inferiority (NI) in progression-free survival (PFS), defined as upper limit of $95 \%$ confidence interval $(\mathrm{CI})$ of hazard ratio $(\mathrm{HR})<1.4$ (first test) and $<1.25$ (second test). Preliminary results presented at the annual meeting of ASCO 2006 showed that 316 patients were enrolled in 13 countries (Table 1). Both arms were well balanced. The primary endpoint was met: capecitabine plus cisplatin was noninferior to fluorouracil plus cisplatin in terms of progression-free survival (HR 0.81 [95\% CI 0.63, 1.04]). The most common treatment-related grade 3/4 AEs (XP vs FP) were: neutropenia (16\% vs 19\%), vomiting ( $7 \%$ vs $9 \%$ ), stomatitis ( $2 \%$ vs $7 \%$ ), diarrhea ( $5 \%$ vs 
Table I Efficacy results of XP vs FP in gastric cancer ${ }^{48}$

\begin{tabular}{llll}
\hline & XP $(\mathbf{n}=160)$ & FP $(\mathbf{n}=156)$ & $\mathbf{P}$ \\
\hline Objective response rate (\%) & 41 & 29 & 0.03 \\
Median progression-free survival (months) & 5.6 & 5.0 & $0.000 I^{*}$ \\
& & & $0.003 * *$ \\
& & $0.10^{* * *}$ \\
Median overall survival (months) & 10.5 & 9.3 & 0.27 \\
\hline
\end{tabular}

"p-value for test of HR vs NI limit of I.4;" similarly with NI limit of I.25; " superiority. Abbreviations: XP, capecitabine/cisplatin; FP, 5-FU/cisplatin.

$5 \%$ ), and anemia ( $5 \%$ vs $3 \%$ ). The rate of all-grade HFS was low $(22 \%$ vs $4 \%)$. These findings suggest that capecitabine should become the fluoropyrimidine of choice for advanced gastric cancer, given the efficacy, reduced hospitalization time and simplified regimen.

In another phase III trial (REAL-2) 1002 patients were randomly assigned to receive triplet therapy with epirubicin and cisplatin plus either fluorouracil (ECF) or capecitabine (ECX) or triplet therapy with epirubicin and oxaliplatin plus either fluorouracil (EOF) or capecitabine (EOX) ${ }^{49}$ The primary end point was noninferiority in OS for the triplet therapies containing capecitabine as compared with fluorouracil and for those containing oxaliplatin as compared with cisplatin. For the capecitabine-fluorouracil comparison, the hazard ratio for death in the capecitabine group was 0.86 (95\% CI, 0.80-0.99); for the oxaliplatin-cisplatin comparison, the HR for the oxaliplatin group was 0.92 (95\% CI, 0.80-1.10). Median survival times in the ECF, ECX, EOF and EOX groups were 9.9 months, 9.9 months, 9.3 months and 11.2 months, respectively; survival rates at 1 year were $37.7 \%, 40.8 \%, 40.4 \%$ and $46.8 \%$, respectively. In the secondary analysis, OS was longer with EOX than with ECF, with a hazard ratio for death of 0.80 in the EOX group (95\% CI, 0.66 to $0.97 ; p=0.02$ ). PFS and response rates did not differ significantly among the regimens. Toxic effects of capecitabine and fluorouracil were similar. Current studies are evaluating the efficacy of capecitabine in combination with other agents, including oxaliplatin as well as in combination with radiation therapy. ${ }^{50}$

\section{Pancreatic cancer}

Initial phase II trials suggested that capecitabine had useful activity in pancreas cancer. A phase II trial by Cartwright et al investigated the safety and efficacy of capecitabine in 42 patients with advanced or metastatic pancreatic cancer. These patients received capecitabine monotherapy (1250 $\mathrm{mg} / \mathrm{m}^{2}$ twice daily on days $1-14$ of a 21 -day cycle) ${ }^{51}$ The safety profile of capecitabine was similar to that observed with capecitabine in colorectal and breast cancer. The major grade $3 / 4$ adverse events were diarrhea, HFS and nausea. There were three confirmed partial responses $(7 \%)$ and a further 17 patients $(41 \%)$ achieved stable disease as their best response (including disease stabilization for 12 weeks in 11 patients [26\%]). The median duration of response was 2.8 months and the median OS was 6.0 months. In addition 10 patients (24\%) achieved a positive Clinical Benefit Response score and pain intensity was reduced in a further 12 patients $(29 \%)$.

As a result of pre-clinical evidence to suggest that capecitabine was synergistic with gemcitabine $\mathrm{e}^{52}$ the combination went on to be tested in clinical trials. A multinational randomized trial by Herrmann et al reported no advantage of adding capecitabine, however subgroup analysis showed the benefit for GemCap in patients with good performance status (HR 0.76; p < 0.03). ${ }^{53}$ Another phase III randomized trial by Cunningham et al that compared single-agent gemcitabine with gemcitabine weekly for 3 weeks plus capecitabine $1660 \mathrm{mg} / \mathrm{m}^{2}$ daily for 21 days every 28-day cycle. ${ }^{54}$ Addition of capecitabine doubled response rate $(14 \%$ vs $7 \% ; \mathrm{p}=0.008)$ and improved OS (HR 0.80; $\mathrm{p}=0.026$ ). Myelosuppression was higher in incidence with the combination arm and HFS was only noted in combination arm. There have been three negative phase III trials of gemcitabine plus a fluoropyrimidine: "Why is this one positive?" The final results of the study are anxiously awaited to answer this question.

Recently, final results of the phase II study of gemcitabine, capecitabine, and bevacizumab in patients with advanced pancreatic cancer have been presented..$^{55}$ Patients received bevacizumab $15 \mathrm{mg} / \mathrm{kg}$ iv day 1 , capecitabine $650 \mathrm{mg} / \mathrm{m}^{2}$ bid days $1-14$, and gemcitabine $1000 \mathrm{mg} / \mathrm{m}^{2}$ iv days 1 and 8; cycles repeated every 21 days. Among 50 patients, 1 patient achieved complete response (CR) (2\%), 10 partial response $(20 \%)$ and 30 stable disease $(60 \%)$. Median PFS and OS were 5.8 and 9.8 months, respectively. Grade 3/4 toxicities included neutropenia $38 \%$, thrombocytopenia $16 \%$, 
thromboembolic events $14 \%$, nausea $14 \%$, hypertension $8 \%$, and gastrointestinal bleeding $8 \%$. One treatment-related death occurred (hemorrhage).

A phase I study of a chemotherapy doublet (gemcitabine plus capecitabine), combined with a biologic doublet (bevacizumab plus erlotinib) in patients with advanced pancreatic adenocarcinoma was presented at the annual meeting of ASCO, 2008 (the TARGET trial). ${ }^{56}$ Patients with advanced (including locally advanced) carcinoma were treated at 4 cohorts of escalating capecitabine doses (days 1-21): $910 \mathrm{mg} / \mathrm{m}^{2}, 1160 \mathrm{mg} / \mathrm{m}^{2}, 1400 \mathrm{mg} / \mathrm{m}^{2}$ and $1660 \mathrm{mg} / \mathrm{m}^{2}$. The doses of co-administered gemcitabine $\left(1000 \mathrm{mg} / \mathrm{m}^{2}\right.$ days 1,8 and 15), bevacizumab (5 mg/kg days 1 and 15), and erlotinib (100 mg/day) every 28 days were constant. Dose-limiting toxicity occurred in 1 patient at $910 \mathrm{mg} / \mathrm{m}^{2}$ (grade 3 epistaxsis) and 2 patients at $1660 \mathrm{mg} / \mathrm{m}^{2}$ (grade 3 diarrhea, and grade 3 skin rash for more than 7 days). No patient developed gastrointestinal perforation or pneumonitis, while a gastrointestinal bleed (grade 1) was seen in 1 patient. Among evaluable 14 patients, there were 5 confirmed partial responses (36\%) and a 50\% decrease in CA 19-9 by 8 weeks was seen in 9 patients (64\%). The maximal tolerable dose of capecitabine in this four-drug cytotoxic/biologic combination is $1660 \mathrm{mg} / \mathrm{m}^{2}$ and a follow-on phase II study is planned.

Kulke et al performed a phase II study of capecitabine plus erlotinib in gemcitabine-refractory advanced pancreatic cancer. ${ }^{57}$ Thirty patients with gemcitabine-refractory metastatic pancreatic cancer were treated with capecitabine, administered at a dose of $1000 \mathrm{mg} / \mathrm{m}^{2}$ twice daily for 2 weeks, followed by a 1 -week break. All patients also received erlotinib $150 \mathrm{mg}$ daily. Treatment with capecitabine and erlotinib in gemcitabine-refractory patients was associated with an overall objective radiologic response rate of $10 \%$ and median survival duration of 6.5 months. In addition, $17 \%$ of the treated patients experienced decreases in tumor marker (CA 19-9) levels of more than 50\% from baseline. Common toxicities included diarrhea, skin rash, fatigue, and HFS. This regimen may offer an acceptable treatment option in patients who experience treatment failure with standard first-line therapy with gemcitabine.

Oral capecitabine has been used in pancreatic cancer as a radio-sensitizing agent. A phase I study done by Saif et al showed that capecitabine $800 \mathrm{mg} / \mathrm{m}^{2}$ bid with concurrent external radiation therapy is feasible in patients with locally advanced pancreatic cancer. ${ }^{58}$ This study was further confirmed by a phase II study. ${ }^{59}$ This approach offers an easy alternative to intravenous fluorouracil as a radiosensitizer but more studies are needed to be done.

\section{Capecitabine in anal cancer}

Since 5-fluorouracil (5-FU) + mitomycin C (MMC)-based chemoradiotherapy is standard treatment for patients with epidermoid anal carcinoma, a recent phase II trial determined the feasibility, toxicity, and efficacy of capecitabine, MMC and radiotherapy (RT) in anal cancer patients. Radiotherapy comprised the schedule of the UK Anal Cancer Trial (ACT) II trial (50.4 Gy in 28 fractions of 1.8 Gy). ${ }^{60}$ With MMC (12 mg/m²) on day 1 and capecitabine on each RT treatment day in 2 divided doses $\left(825 \mathrm{mg} / \mathrm{m}^{2}\right.$ bid). The endpoints were CR at 4 weeks, local control at 6 months and toxicity.

Thirty-one patients entered the trial. The median age was 61 years (range 45-86) with 14 males and 17 females. Compliance with chemotherapy with no dose interruptions or delays was $68 \%$, and with RT was $81 \%$. Eighteen (58\%) patients completed both modalities of treatment as planned. Dose-limiting Grade 3 or 4 diarrhea was seen in 1 of 31 patients. Three patients experienced Grade 3 neutropenia. There were no treatment-related deaths. Four weeks following completion of chemoradiation, 24 patients (77\%) had a complete clinical response and $4(16 \%)$ a partial response. With a median follow-up of 14 months, 3 locoregional relapses occurred.

Capecitabine with MMC and RT in patients with anal carcinoma is well tolerated, with minimal toxicity and acceptable compliance. Further testing of this schedule in Phase III studies is undergoing.

\section{Capecitabine in hepatobiliary cancers}

Capecitabine is also actively in hepatobiliary tumors. Patt et al performed a retrospective analysis of all patients with hepatocellular carcinoma (HCC), cholangiocarcinoma (CCA), or gallbladder carcinoma (GBC) who were treated with oral capecitabine. ${ }^{61}$ A total of 63 patients were treated with capecitabine ( 37 with $\mathrm{HCC}, 18$ with CCA, 8 with GBC). Capecitabine $1000 \mathrm{mg} / \mathrm{m}^{2}$ was administered twice daily for 14 days. Treatment was repeated every 21 days. Each patient received 1 to 15 treatment cycles. Nine (14\%) to $11 \%$ of patients with $\mathrm{HCC}, 6 \%$ of patients with $\mathrm{CCA}$, and $50 \%$ of patients with $\mathrm{GBC}$ had either a $\mathrm{CR}$ or a partial response. A CR was radiologically confirmed in 1 patient with $\mathrm{HCC}$ and in 2 patients with GBC. The median survival times were 10.1 months (95\% CI 4.5-15.7 months) for patients with HCC, 8.1 months (95\% CI, 7.4-8.9 months) for patients with CCA, and 9.9 months (95\% CI, 4.4-15.4 months) for patients with GBC. The most common toxicity was HFS (37\%). Grade 3 thrombocytopenia occurred in $8 \%$ of patients with $\mathrm{HCC}$. 
No other significant toxicities were observed. For all patients, response to treatment was positively correlated with survival and decline in tumor markers. The author concluded that capecitabine is safe for patients with hepatobiliary carcinoma, including those with cirrhosis. The antitumor activity of single-agent capecitabine was most pronounced in patients with GBC, was modest in patients with $\mathrm{HCC}$, and was poor in patients with CCA.

The combination of capecitabine with platinum compounds as well as triplet has also been studied in small studies. ${ }^{62-64}$ Worth-mentioning studies may include but are not limited to the FFCD 03-03 trial: a multicenter phase II trial of capecitabine plus oxaliplatin (XELOX); capecitabine plus cisplatin; and triplet of doxorubicin, cisplatin and capecitabine for metastatic hepatocellular carcinoma.

\section{Discussion}

Capecitabine is currently the only novel, orally homeadministered fluorouracil prodrug. Capecitabine could replace 5-FU either as a single agent or as the combination partner for chemotherapy or radiotherapy in nearly all gastrointestinal malignancies. It offers patients more freedom from hospital visits and less inconvenience and complications associated with infusion devices. The drug has been extensively studied in large clinical trials in many gastrointestinal and non-gastrointestinal malignancies, and compares favorably with fluorouracil with a safe toxicity profile, consisting mainly of gastrointestinal and dermatologic adverse effects. Whereas gastrointestinal events and HFS occur often with capecitabine, the tolerability profile is comparatively favorable. Prompt recognition of severe adverse effects is the key to successful management of capecitabine.

Ongoing and future clinical trials will continue to examine, and likely expand, the role of capecitabine as a single agent and/or in combination with other anticancer agents for the treatment of other solid tumors, both in the advanced palliative and adjuvant settings.

It is important to appreciate that the combination of capecitabine with agents such as irinotecan and oxaliplatin is more than just replacing the backbone of 5-FU. There is a molecular rationale: XELIRI $\rightarrow$ capecitabine inhibits $\mathrm{Bcl}-2$, a protein that prevents tumor cell death (apoptosis) by radiation, taxanes, oxaliplatin and irinotecan, and $\mathrm{XELOX} \rightarrow$ capecitabine inhibits ERCC-1, a DNA repair protein that blocks the cytotoxic activity of radiation and oxaliplatin. In addition, irinotecan and oxaliplatin induce TP in tumors. Similarly, our group has also investigated the effect of radiation on TP and showed that radiation upregulates TP in tumors, but not in normal cells. This combined modality then synergizes in both directions: radiation as chemosensitizer by upregulating TP and capecitabine after conversion to 5-FU radiosensitizing the tumor to radiation effects. This combination is of utmost interest in clinical investigation in many tumors, especially rectal cancer.

It is also important to appreciate that dose reductions in pivotal studies in colorectal cancer indicated that dose reduction did not reduce the efficacy of capecitabine. ${ }^{65}$ Currently, $2500 \mathrm{mg} / \mathrm{m}^{2}$ is the FDA-approved dose of capecitabine but this dose is not tolerated by patients in the US. Another issue related to the dose is the two different strengths of capecitabine ( 150 and $500 \mathrm{mg}$ ). Some physicians prefer to prescribe the dose with $500 \mathrm{mg}$ capsules only to prevent any overdose or underdose by a patient. We also suggest that the dose should be rounded to the closest figure to make a dose that contains a similar strength of capecitabine capsules for convenience and to decrease the risk as noted above.

One interesting observation was recently published by Hennig et al ${ }^{66}$ who compared overall acceptability to patients between intravenous FU/LV or oral capecitabine. Patients scheduled for adjuvant single-agent fluoropyrimidine therapy were randomly assigned to receive once-weekly FU/LV ( $425 \mathrm{mg} / \mathrm{m}^{2} \mathrm{FU}, 45 \mathrm{mg} \mathrm{LV}$ ) for 6 weeks, followed by two 3 -week cycles of capecitabine $\left(1250 \mathrm{mg} / \mathrm{m}^{2}\right.$ twice daily, days 1 through 14), or the same treatments but in reverse order. After 12 weeks, the patients were asked which treatment they preferred, and received the preferred treatment for an additional 12 weeks. The primary end point was patient preference. After 40 of the planned 74 patients had been randomly assigned, real-time adverse event monitoring led to early trial closure because of excess sequence-specific toxicity. In chemotherapy-naïve patients, capecitabine produced more toxicity than FU/LV, but at levels in line with previously reported data. However, treatment with capecitabine after FU/LV caused markedly increased toxicity, indicating a sequence-specific interaction as shown in Table 2. The mechanism has not been determined, but interaction with intracellularly retained folate after FU/LV therapy is a possibility. Oncologists need to be aware of this risk if considering crossing patients over from FU/LV to capecitabine-based regimens.

Education of the patient and the caretaker is paramount in management of capecitabine toxicity. There are several drug interactions with capecitabine and the patient must be educated to prevent adverse outcomes. Data on patients with DPD deficiency and tolerance of capecitabine are scarce. ${ }^{67}$ We suggest that it is still important to suspect and 
Table 2 Grade $\geq 3$ toxicity experienced and dose adjustments due to toxicity in the PACT trial safety population, comparing start and switch periods

\begin{tabular}{|c|c|c|c|c|c|c|}
\hline \multirow[t]{2}{*}{ Characteristic } & \multicolumn{3}{|l|}{ Capecitabine } & \multicolumn{3}{|l|}{ FU/LV } \\
\hline & No of patients & $\%$ & $95 \% \mathrm{Cl}$ & No of patients & $\%$ & $95 \% \mathrm{Cl}$ \\
\hline \multicolumn{7}{|l|}{ Grade $\geq 3$ toxicity } \\
\hline Given in start period & 5 of 18 & 28 & & 0 of 16 & 0 & \\
\hline Given in switch period & II of I4 & 79 & & 0 of 12 & 0 & \\
\hline Difference & & 51 & 15 to 87 & 0 & 0 & NA \\
\hline \multicolumn{7}{|l|}{ Dose delay or stoppage } \\
\hline Given in start period & 8 of 18 & 44 & & I of 16 & 6 & \\
\hline Given in switch period & 12 of 14 & 86 & & 0 of 12 & 0 & \\
\hline Difference & & 41 & 6 to 77 & & -6 & -26 to 13 \\
\hline
\end{tabular}

Abbreviations: PACT, Patient Preference in Adjuvant Colorectal Therapy trial; FU/LV, fluorouracil with leucovorin; NA, not applicable.

test for DPD deficiency in patients on capecitabine with any manifestation of toxicity.

\section{Summary}

In summary, most current data indicate that capecitabine is not inferior to infusional 5-FU and offers added benefit of convenience. Future studies should also aim at evaluating the drug cost. For those parts of the world in which hospitalization is required to deliver fluorouracil by infusion, an outpatient oral regimen has an advantage. Capecitabine offers not only efficacy in different gastrointestinal and non-gastrointestinal malignancies, but also broadens the availability and convenience of treatment.

\section{Acknowldgments}

I thank Sharon Gould to help me in preparing this manuscript.

\section{Disclosures}

The author has no conflicts of interest to disclose.

\section{References}

1. Miwa M, Ura M, Nishida M, et al. Design of a novel oral fluoropyrimidine carbamate, capecitabine, which generates 5-fluorouracil selectively in tumours by enzymes concentrated in human liver and cancer tissue. Eur J Cancer. 1998;34:1274-1281.

2. Ishikawa T, Utoh M, Sawada N, et al. Tumor selective delivery of 5-fluorouracil by capecitabine, a new oral fluoropyrimidine carbamate, in human cancer xenografts. Biochem Pharmacol. 1998;55(7): 1091-1097.

3. Schuller, J, Cassidy, J, Dumont, E, et al. Preferential activation of capecitabine in tumor following oral administration to colorectal cancer patients. Cancer Chemother Pharmacol. 2000;45:291.

4. Reigner B, Blesch K, Weidekaam E. Capecitabine; a review of its clinical pharmacokinetics. Clin Pharmacokinet. 2001;40:85-104.

5. Nakayama Y, Inoue Y, Nagashima N, et al. Expression levels of thymidine phosphorylase (TP) and dihydropyrimidine dehydrogenase (DPD) in patients with gastrointestinal cancer. Anticancer Res. 2005;25(6A):3755-3761.
6. Judson I, Beale P, Trigo J, et al. A human capecitabine excretion balance and pharmacokinetic study after administration of asingle oral dose of 14C-labelled drug. Invest New Drugs. 1999;17:49-56.

7. Twelves C, Glynne-Jones R, Cassidy J, et al. Effect of hepatic dysfunction due to liver metastases on the pharmacokinetics of capecitabine and its metabolites. Clin Cancer Res. 1999;5:1696-1702.

8. Poole $\mathrm{C}$, et al. Effect of renal impairment on the pharmacokinetics and tolerability of capecitabine (Xeloda) in cancer patients. Cancer Chemother Pharmacol. 2002;49:225-234.

9. Saif MW. An adverse interaction between warfarin and fluoropyrimidines revisited. Clin Colorectal Cancer. 2005;5(3):175-180.

10. Reigner B, Clive S, Cassidy J, et al. Influence of the antacid maalox on the pharmacokinetics of capecitabine in cancer patients. Cancer Chemother Pharmacol. 1999;43:309-315.

11. Budman DR, Meropol NJ, Reigner B. Preliminary studies of a novel oral fluoropyrimidine carbamate: capecitabine. J Clin Oncol. 1998;16:1795.

12. Saif MW, Tomita M, Ledbetter L, Diasio RB. Capecitabine-related cardiotoxicity: recognition and management. J Support Oncol. 2008;6(1):41-48.

13. Saif MW, Katirtzoglou NA, Syrigos KN. Capecitabine: an overview of the side effects and their management. Anticancer Drugs. 2008;19(5):447-464.

14. Van Cutsem E, Hoff PM, Harper P, et al. Oral capecitabine vs iv 5-FU and LV: integrated efficacy data and novel analyses from two large, randomized phase III trials. Br J Cancer. 2004;90: 1190-1197.

15. Kim TW, Kang WK, Chang HM, et al. Multicenter phase II study of oral capecitabine plus irinotecan as first-line chemotherapy in advanced colorectal cancer: a Korean cancer study group trial. Acta Oncol. 2005;44:230-235.

16. Bajetta E, Bartolomeo MD, Mariani L, et al. Randomized multicenter phase II of two different schedules of irinotecan combined with capecitabine as first-line treatment in metastatic colorectal carcinoma. Cancer. 2004;100:279-287.

17. Cassidy J, Tabernero J, Twelves C, et al. Xelox (capecitabine plus oxilaplatin): active first-line therapy for patients with metastatic colorectal cancer. J Clin Oncol. 2004;22:2084-2091.

18. Zeuli M, Nardoni C, Pino MS, et al. Phase II study of capecitabine and oxaliplatin as first-line treatment in advanced colorectal cancer. Ann Oncol. 2003;14:1378-1382.

19. Borner MM, Dietrich D, Stupp R, et al. Phase II study of capecitabine and oxaliplatin in first- and second-line treatment of advanced or metastatic colorectal cancer. J Clin Oncol. 2002;20:1759-1766.

20. Shields AF, Zalupski MM, Marshall JL, Meropol NJ. Treatment of advanced colorectal carcinoma with oxaliplatin and capecitabine. Cancer. 2003;100:531-537. 
21. Scheithauer W, McKendrick J, Begdie S, et al. Oral capecitabine as an alternative to iv 5-FU-based adjuvant therapy for colon cancer: safety results of a randomized phase III trial. Ann Oncol. 2003;14:1735-1743.

22. Lamberti C, Sauerbruch T, Glasmacher A. Adjuvant capecitabine is at least as effective as fluorouracil plus leucovorin for survival in people with resected stage III colon cancer. Cancer Treat Rev. 2005;31:648-652.

23. Twelves C, Wong A, Nowacki MP, Abt M, Burris H III, Carrato A, et al. Capecitabine as adjuvant treatment for stage III colon cancer. N Engl Med. 2005;352:2696-2704.

24. Twelves C, Boyer M, Findlay M, et al. Capecitabine (Xeloda) improves medical resource use compared with 5-FU plus LV in a phase III trial conducted in patients with advanced colorectal carcinoma. Eur J Cancer. 2001;37:597-604.

25. Cassidy J, Twelves C, Van Cutsem E, Hoff P, Bajetta E, Boyer M, et al. First-line oral capecitabine therapy in metastatic colorectal cancer: a favorable safety profile compared with intravenous 5-FU/LV. Ann Oncol. 2002;13:566-575.

26. Twelves C, Boyer M, Findlay M, et al; Xeloda Colorectal Cancer Study Group. Capecitabine (Xeloda) improves medical resource use compared with 5-fluorouracil plus leucovorin in a phase III trial conducted in patients with advanced colorectal carcinoma. Eur J Cancer. 2001;37(5):597-604.

27. Cassidy J, Clarke S, Díaz-Rubio E, et al. Randomized phase III study of capecitabine plus oxaliplatin compared with fluorouracil/folinic acid plus oxaliplatin as first-line therapy for metastatic colorectal cancer. J Clin Oncol. 2008;26(12):2006-2012.

28. Saltz LB, Clarke S, Díaz-Rubio E, et al. Bevacizumab in combination with oxaliplatin-based chemotherapy as first-line therapy in metastatic colorectal cancer: a randomized phase III study. J Clin Oncol. 2008;26(12):2013-2019.

29. Gennatas C, Michalaki V, Gennatas S, Papalambros E. Irinotecan plus capecitabine as first-line chemotherapy in advanced colorectal cancer. Anticancer Res. 2008;28(3B):1923-1926.

30. Fuchs CS, Marshall J, Barrueco J. Randomized, controlled trial of irinotecan plus infusional, bolus, or oral fluoropyrimidines in first-line treatment of metastatic colorectal cancer: updated results from the BICC-C study. J Clin Oncol. 2008;26(4):689-690.

31. Rea DW, Nortier JWR, Ten Bokkel Huinink WW, et al. A phase I/II and pharmacokinetic study of irinotecan in combination with capecitabine as first-line therapy for advanced colorectal cancer. Ann Oncol. 2005;16(7):1123-1132.

32. Douillard JY, Cunningham $\mathrm{D}$, Roth $\mathrm{AD}$, et al. Irinotecan combined with fluorouracil compared with fluorouracil alone as first-line treatment for metastatic colorectal cancer: a multicenter randomized trial. Lancet. 2000;355:1041-1047.

33. Twelves C, Scheithauer W, McKendrick J, et al. Capecitabine versus 5-FU/LV in stage III colon cancer: Updated 5-year efficacy data from $\mathrm{X}$-ACT trial and preliminary analysis of relationship between hand-foot syndrome (HFS) and efficacy. Gastrointestinal Cancers Symposium. 2008; Abstr. 274.

34. Schmoll HJ, Tabernero J, Nowacki M. Early safety findings from a phase III trial of capecitabine plus oxaliplatin (XELOX) vs bolus 5-FU/LV as adjuvant therapy for patients (pts) with stage III colon cancer. Proc Am Soc Clin Oncol. 2005; Abstr. 3523.

35. Andre T, Boni C, Mounedji-Boudiaf L, et al. Oxaliplatin, fluorouracil and leucovorin as adjuvant treatment for colon cancer. $N$ Engl Med. 2004;350:2343-2351.

36. Dunst J, Reese T, Debus J. Phase-II study of preoperative chemoradiation with capecitabine in rectal cancer. Proc Am Soc Clin Oncol. 2004; 23 : Abstr. 3559

37. Lin EH, Skibber J, Delcos M. Phase II study of capecitabine and concomitant boost radiotherapy (XRT) in patients (pts) with locally advanced rectal cancer (LARC). J Clin Oncol. 2005;(23 Suppl 16s):Abstr. 3593.

38. Dupuis O, Vie B, Liedo G. Capecitabine (X) chemoradiation (CRT) in the preoperative treatment of patients (pts) with rectal adenocarcinomas: a phase II GERCOR trial. Proc Am Soc Clin Oncol. 2004;23: Abstr. 3538
39. Krishnan S, Janjan NA, Skibber JM, Rodriguez-Bigas MA, Wolff RA, Das P, et al. Phase II study of capecitabine (Xeloda) and concomitant boost radiotherapy in patients with locally advanced rectal cancer. Int J Radiat Oncol Biol Phys. 2006;66:762-771.

40. Kim JC, Kim TW, Kim JH, et al. Preoperative concurrent radiotherapy with capecitabine before total mesorectal excision in locally advanced rectal cancer. Int $J$ Radiat Oncol Biol Phys. 2005;63:346-353.

41. Saif MW, Hasmi S, Zelterman D, Almhanna K, Kim R. Capecitabine vs continuous infusion 5-FU in neoadjuvant treatment of rectal cancer. Int J Colorectal Dis. 2008;23(2):139-145.

42. Machiels JP, Duck L, Honhon B, et al. Phase II study of preoperative oxaliplatin, capecitabine and external beam radiotherapy in patients with rectal cancer: the RadiOxCape study. Ann Oncol. 2005;16:1898-1805.

43. Rodel C, Liersch T, Hermann RM, et al. Multicenter phase II trial of chemoradiation with oxaliplatin for rectal cancer. J Clin Oncol. 2007:25:110-117.

44. Rodel C, Grabenbauer GG, Papadopoulos T, et al. Phase I/II trial of capecitabine, oxaliplatin and radiation for rectal cancer. J Clin Oncol. 2003;21:3098-3004.

45. Machiels JPP, Duck L, Honhon B. Phase II study of preoperative oxaliplatin, capecitabine and external beam radiotherapy in patients with locally advanced rectal adenocarcinomas: the RadiOxCape Study. Proc Am Soc Clin Oncol G1. 2005:Abstr. 245.

46. Willeke F, Tiefenbacher U, Hochhaus A. Phase II trial of capecitabine and irotecan in combination with concurrent radiotherapy for neoadjuvant treatment of locally advanced rectal cancer. J Clin Oncol. 2005;(23 Suppl 16S):268s. Abstr. 3589.

47. Gollins SW, Myint S, Levine E. Phase I study of radiotherapy (RT) plus concurrent irinotecan (CPT-11) and capecitabine (CAP) as preoperative downstaging treatment for locally advanced inoperable rectal cancer. J Clin Oncol. 2005;(23 Suppl 16S):262s (Abstr. 3565).

48. Kang Y, Kang WK, Shin DB, et al. Randomized phase III trial of capecitabine/cisplatin (XP) vs continuous infusion of 5-FU/cisplatin (FP) as first-line therapy in patients (pts) with advanced gastric cancer (AGC): efficacy and safety results. J Clin Oncol. 2006 ASCO Annual Meeting Proceedings Part I. Vol 24, No. 18S (June 20 Suppl), 2006; LBA4018.

49. Cunningham D, Cunningham D, Starling N; Upper Gastrointestinal Clinical Studies Group of the National Cancer Research Institute of the United Kingdom. Capecitabine and oxaliplatin for advanced esophagogastric cancer. $N$ Engl J Med. 2008;358(1):36-46.

50. Hofheinz RD, Wenz F, Lukan N, et al. Oxaliplatin and capecitabinebased chemoradiotherapy for gastric cancer-An extended phase I MARGIT and AIO Trial. Int J Radiat Oncol Biol Phys. 2008;Jun 6.

51. Cartwright TH, Cohn A, Varkey JA, Chen YM, Szatrowski TP, Cox JV, Schulz JJ. Phase II study of oral capecitabine in patients with advanced or metastatic pancreatic cancer. J Clin Oncol. 2002;20(1): 160-164.

52. Saeki T, Takashima S. Mechanism and possible biochemical modulation of capecitabine (Xeloda), a newly generated oral fluoropyrimidine. Gan To Kagaku Ryoho. 1999;26:447-455.

53. Herrmann R, Bodoky G, Ruhstaller T, Glimelius B, Bajetta E, Schüller J, et al. Gemcitabine plus capecitabine compared with gemcitabine alone in advanced pancreatic cancer: a randomized, multicenter, phase III trial of the Swiss Group for Clinical Cancer Research and the Central European Cooperative Oncology Group. J Clin Oncol. 2007;25:2212-2217.

54. Cunningham D, Chau I, Stocken C, et al. Phase III randomized comparison of gemcitabine (GEM) versus gemcitabine plus capecitabine (GEM-CAP) in patients with advanced pancreatic cancer. Eur J Cancer Suppl. 2005;3:12. Abstr. PS11.

55. Iyer RV, Yu J, Garrett CR, Litwin AM, Kuvshinoff B, Tarquini M, et al. Gemcitabine, capecitabine, and bevacizumab in patients with advanced pancreatic cancer (APC): Final results of the multicenter phase II study. Gastrointestinal Cancers Symposium. 2008; Abstr. 198. 
56. Starling N, Watkins D, Chau I, Norman A, Fairhead E, Thomas J, et al. A phase I study of a chemotherapy doublet (gemcitabine plus capecitabine [GemCap]), combined with a biologic doublet (bevacizumab plus erlotinib) in patients with advanced pancreatic adenocarcinoma (PC): The TARGET Trial. 2008 Gastrointestinal Cancers Symposium. Abstr. 141.

57. Kulke MH, Blaszkowsky LS, Ryan DP, et al. Capecitabine plus erlotinib in gemcitabine-refractory advanced pancreatic cancer. J Clin Oncol. 2007;25(30):4787-4792.

58. Saif MW, Eloubeidi MA, Russo S, et al. Phase I study of capecitabine with concomitant radiotherapy for patients with locally advanced pancreatic cancer: expression analysis of genes related to outcome. J Clin Oncol. 2005;23:8679-8687.

59. Saif MW, Black G, Roy S, et al. Phase II study of capecitabine with concomitant radiotherapy for patients with locally advanced pancreatic cancer: up-regulation of thymidine phosphorylase. Cancer J. 2007;13(4):247-256.

60. Glynne-Jones R, Meadows H, Wan S, et al. National Cancer Research Institute Anal Sub Group and Colorectal Clinical Oncology Group. EXTRA - a multicenter phase II study of chemoradiation using a 5 day per week oral regimen of capecitabine and intravenous mitomycin $\mathrm{C}$ in anal cancer. Int J Radiat Oncol Biol Phys. 2008;72(1):119-126.
61. Patt YZ, Hassan MM, Aguayo A, et al. Oral capecitabine for the treatment of hepatocellular carcinoma, cholangiocarcinoma, and gallbladder carcinoma. Cancer. 2004;101(3):578-586.

62. Shim JH, Park JW, Nam BH, Lee WJ, Kim CM. Efficacy of combination chemotherapy with capecitabine plus cisplatin in patients with unresectable hepatocellular carcinoma. Cancer Chemother Pharmacol. 2008; Apr 25.

63. Boige V, Raoul JL, Pignon JP; Fédération Francophone de Cancérologie Digestive. Multicentre phase II trial of capecitabine plus oxaliplatin (XELOX) in patients with advanced hepatocellular carcinoma: FFCD 03-03 trial. Br J Cancer. 2007;97(7):862-867.

64. Park SH, Lee Y, Han SH, et al. Systemic chemotherapy with doxorubicin, cisplatin and capecitabine for metastatic hepatocellular carcinoma. BMC Cancer. 2006;6:3.

65. Jonker DJ, Vincent M, Kerr I. Dose reduced first-line capecitabine monotherapy in older and less fit patients with advanced colorectal cancer. Proc Am Soc Clin Oncol GI. 2004; Abstr. 212.

66. Hennig IM, Naik JD, Brown S, et al. Severe sequence-specific toxicity when capecitabine is given after Fluorouracil and leucovorin. J Clin Oncol. 2008;26(20):3411-3417.

67. Saif MW, Diasio R. Is capecitabine safe in patients with gastrointestinal cancer and dihydropyrimidine dehydrogenase deficiency? Clin Colorectal Cancer. 2006;(5):359-362. 
